\title{
THE DEVELOPMENT OF ROLLING TEXTURES IN LOW-CARBON STEELS
}

\author{
H. INAGAKI and T. SUDA \\ Technical Research Center, Nippon Kokan Co. Ltd., 210 Kawasaki-shi, Japan
}

(Received June 1, 1972)

\begin{abstract}
The crystallite orientation distribution analysis was applied to the study of the development of the rolling textures in low-carbon steels. It was found that the constraining effect of the grain boundary remarkably influences the rolling textures of polycrystalline iron. This enhances the crystal rotations, which would not be expected to occur in single crystals; and grains having the $\{111\}\langle 112\rangle$ orientations are forced to rotate about the $\langle 111\rangle$ axes lying in the sheet normal direction toward the $\{111\}\langle 110\rangle$ orientations. This is the origin of the $\langle 111\rangle$ fiber texture normally found in the rolling textures of low-carbon steels. The presence of the partial fiber texture having the $\langle 110\rangle$ axes inclined $30 \mathrm{deg}$ from the sheet normal toward the rolling direction could not be confirmed.
\end{abstract}

\section{INTRODUCTION}

The rolling textures of polycrystalline iron have been described in various ways. ${ }^{1-9}$ According to Barrett and Levenson, ${ }^{1}$ these consist of two continuous sets of end orientations. The first set is a partial fiber texture with the $\langle 110\rangle$ fiber axes in the rolling direction, while the second set is a fiber texture having the $\langle 111\rangle$ axes in the sheet normal direction. The presence of the first set has been confirmed by all investigators. As to the second set, there have been controversies in its description.

Based on the (110) pole figures of cold rolled carbonyl iron, Haessner and Weik ${ }^{2}$ described this in terms of a $\langle 110\rangle$ fiber texture having the $\langle 110\rangle$ axes inclined $35 \mathrm{deg}$ from the sheet normal toward the rolling direction.

Bennewitz ${ }^{4}$ found, on the other hand, that the second set of orientations could better be described as a partial fiber texture with the $\langle 110\rangle$ axes inclined $30 \mathrm{deg}$ from the sheet normal toward the rolling direction. This was designated as a " $\langle 110\rangle$ 60 deg RD fiber texture". On this basis, he divided the development of the rolling texture into three stages.

However, the presence of the " $\langle 110\rangle 60 \mathrm{deg}$ RD fiber axes" is theoretically difficult to explain. Direct experimental evidence, verifying that individual grains really rotate about these fiber axes, have not been obtained so far. The presence of these fiber axes was only indirectly inferred from the pole figures, above all from (110) pole figures.
Generally, pole figures describe only the distribution of particular crystallographic axes in the sample coordinate system. Two crystallites, whose orientations are related to each other by a rotation about one of these axes cannot be discriminated in the pole figure. In this respect, the precise distribution of the crystallite around the $\langle 110\rangle$ fiber axes cannot adequately be described by the (110) pole figure alone. This uncertainty is completely eliminated in the method of the crystallite orientation distribution analysis developed by $\mathrm{Roe}^{10-13}$ and Bunge ${ }^{14-18}$.

It is the purpose of the present investigation, using this method of analysis, to study the formation of the rolling textures in low-carbon steels. For the detailed discussions on the formation of the fiber textures, it is necessary to clarify how individual grains reorient during cold rolling. To this end, several polycrystalline specimens with sharp initial textures were cold rolled, and from their orientation changes the behaviour of a grain having a specific initial orientation in a random polycrystal was indirectly deduced. The results were finally compared with the development of the rolling texture observed in a low-carbon steel having a weak initial texture.

\section{EXPERIMENTAL PROCEDURE AND METHOD OF ANALYSIS}

Chemical compositions and hot rolling finishing and coiling temperatures of the starting hot bands 
TABLE I

Chemical composition and hot rolling temperatures

\begin{tabular}{|c|c|c|c|c|c|c|c|c|}
\hline \multirow{2}{*}{ Steel } & \multirow{2}{*}{$\mathrm{C}$} & \multirow{2}{*}{$\mathrm{Mn}$} & \multirow{2}{*}{$\mathbf{P}$} & \multirow{2}{*}{$\mathbf{S}$} & \multirow{2}{*}{$\mathbf{N}$} & \multirow{2}{*}{ sol'Al } & \multicolumn{2}{|c|}{ Rolling temp. } \\
\hline & & & & & & & Finish, ${ }^{\circ} \mathrm{C}$ & Coil, ${ }^{\circ} \mathrm{C}$ \\
\hline 1 & 0.005 & 0.41 & 0.011 & 0.015 & 0.0024 & - & 890 & 600 \\
\hline 4 & 0.056 & 0.29 & 0.012 & 0.029 & 0.0019 & - & 760 & 660 \\
\hline 13 & 0.054 & 0.29 & 0.009 & 0.022 & 0.0056 & 0.029 & 865 & 530 \\
\hline 18 & 0.005 & 0.0005 & 0.002 & 0.006 & 0.0012 & 0.001 & 1000 & Air cooled \\
\hline
\end{tabular}

are given in Table I. Steels 1 and 4 are rimmed steels, while Steel 13 is an aluminum killed steel. Steel 18 is an experimentally hot rolled electrolytic iron.

Sharp initial textures were obtained from these steels in the following way.

(1) $\{110\}\langle 001\rangle$ texture: Hot bands from Steel 1 were held $5 \mathrm{~min}$ at $1250^{\circ} \mathrm{C}$ and cooled in air. After 70 pct cold reduction, specimens were annealed for $1 \mathrm{hr}$ at $750^{\circ} \mathrm{C}$.

(2) $\{110\}\langle 110\rangle$ texture: The transverse direction of the $\{110\}\langle 001\rangle$ specimen was chosen as the rolling direction.

(3) $\{001\}\langle 110\rangle$ texture: Hot bands from Steel 4 were used in the as-received condition.

(4) $\{111\}\langle 110\rangle$ texture: Hot bands from Steel 13 were cold rolled 70 pct. Specimens were then heated to $700^{\circ} \mathrm{C}$ with a heating rate of $20 \mathrm{deg} / \mathrm{hr}$ and held $3 \mathrm{hrs}$. at $700^{\circ} \mathrm{C}$.

(5) $\{111\}\langle 112\rangle$ texture: The transverse direction of the $\{111\}\langle 110\rangle$ specimen was chosen as the rolling direction.

These specimens were finally cold rolled under good lubrications up to 95 pct reduction in thickness.

The crystal rotation produced by cold rolling was observed by revealing etch pits on the rolling planes of Steel 18, cold rolled 70 pct. Specimens were ground to the center of their thickness and (001) facet pits were produced. ${ }^{19}$ A JEOL-JSM-U3 scanning electron microscope was used for the observation.

Samples for texture determinations were chemically thinned to about $0.04 \mathrm{~mm}$ thickness. Using both the transmission ${ }^{20}$ and reflection ${ }^{21}$ technique, complete (200), (110) and (211) pole figures were determined. After various corrections were made normalized intensities were obtained. ${ }^{22}$
The crystallite orientation distribution analysis was made with Roe's method. ${ }^{10-13,23}$ The crystallite orientation distribution is usually expanded in a series of generalized spherical harmonics. In the present study, the series expansion was truncated at $22 \mathrm{nd}$ order.

The crystallite orientation distribution takes often, in practice, negative value. As this is physically meaningless, the largest negative value on the $\phi=45 \mathrm{deg}$ section was regarded, in the present study, as the total possible error. This was designated by Nand shown aboveeach set of figures, which will be shown later. All the calculations were
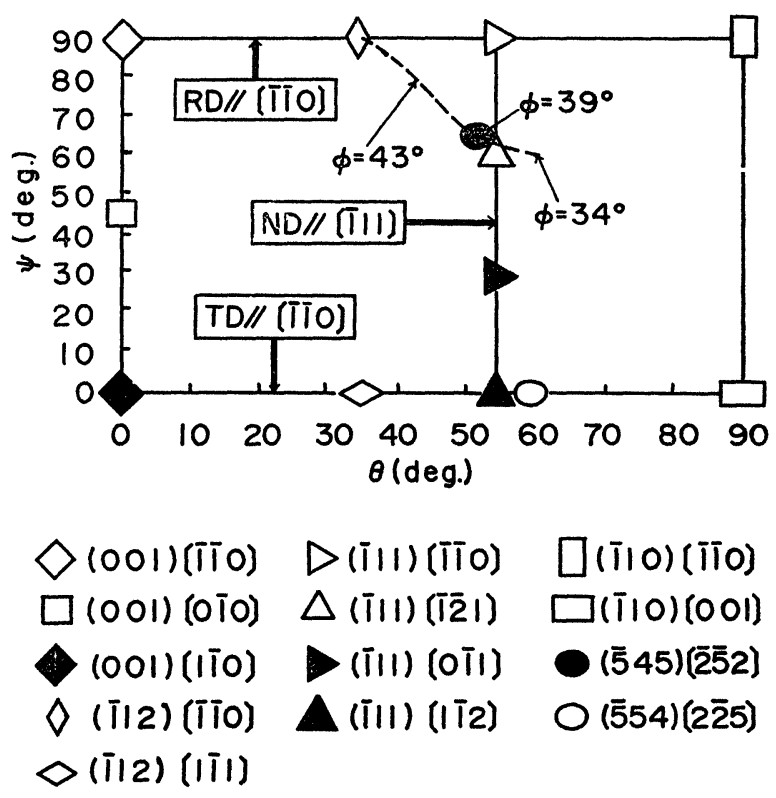

---- (TOI) $60^{\circ}$ from RD

FIGURE 1 The locations of ideal orientations on the $\phi=45 \mathrm{deg}$ section of the crystallite orientation distribution. The positions of important fiber textures are also shown. (See text). 
carried out using an IBM 360-65 computer. The program was so constructed that the $\phi=45 \mathrm{deg}$ section and constant $\psi$ sections of the crystallite orientation distribution appeared directly on a line printer.

The locations of ideal orientations on the $\phi=45 \mathrm{deg}$ section are shown in Figure 1. Orientations having the $\langle 110\rangle$ axes in the rolling direction are located on the $\psi=90 \mathrm{deg}$ line (indicated by $\mathrm{RD} \|[\overline{1} \overline{1} 0])$, while those having the $\langle 111\rangle$ axes in the sheet normal direction are situated on the $\theta=55$ deg line (indicated by ND\| [111]). Orientations having the $\langle 110\rangle$ directions in the transverse direction appear on the $\psi=0$ deg line (indicated by $\mathrm{TD} \|[\overline{1} \overline{1} 0])$. Locations of the members of the $\langle 110\rangle 60 \mathrm{deg} \mathrm{RD}$ fiber textures are, in the range $\phi=34$ to $45 \mathrm{deg}$, projected on the $\phi=45 \mathrm{deg}$ section and are shown in dashed line (indicated by [101] $60^{\circ}$ from RD). For orthotropic physical symmetry and cubic crystal symmetry, the crystallite orientation distribution takes the same value for each member of the family $\{h k l\}\langle u v w\rangle$. In the present study, the latter notation is adopted.

\section{RESULTS AND DISCUSSIONS}

The $\{111\}\langle 110\rangle$ specimen Figure $2(a)$ is the $\phi=45 \mathrm{deg}$ section of the crystallite orientation distribution, showing the $\{111\}\langle 110\rangle$ initial texture, while Figures 2(b) and (c) are $\phi=45 \mathrm{deg}$ sections, showing the rolling textures of this specimen at 70 and 85 pct reductions, respectively.

After 70 pct reduction, a high intensity region developed uniformly along the $\theta=55 \mathrm{deg}$ line, Figure 2(b). This indicates the formation of the $\langle 111\rangle$ fiber texture having the $\langle 111\rangle$ fiber axes in the sheet normal direction. The $\{111\}\langle 110\rangle$ initial texture merged into this $\langle 111\rangle$ fiber texture. (The origin of the $\langle 111\rangle$ fiber texture seems to be found in the $\{554\}\langle 225\rangle$ and $\{111\}\langle 112\rangle$ orientations, which were present in a sufficient amount in the orientation spread of the $\{111\}\langle 110\rangle$ initial texture. Evidence in support of this possibility will be given later.)

After 85 pct reduction, this $\langle 111\rangle$ fiber texture did not develop further, Figure 2(c). At this stage the $\{111\}\langle 110\rangle$ components increased remarkably and their presence became evident again on the $\theta=55$ deg line.

These intensity changes of the $\{111\}\langle 110\rangle$ orientations suggest that grains having the $\{111\}$ $\langle 110\rangle$ initial orientations were stable up to $85 \mathrm{pct}$ reduction in thickness.
To explain the increase of the $\{111\}\langle 110\rangle$ orientations observed above 70 pct reduction, it would be necessary to assume that some part of the $\{111\}\langle 110\rangle$ orientations were formed from the $\langle 111\rangle$ fiber texture.

It is to be noted in Figure 2(c) that the exact position of the maximum on the $\psi=90 \mathrm{deg}$ line is not at $\theta=55 \mathrm{deg}$, but at $\theta=50 \mathrm{deg}$. This
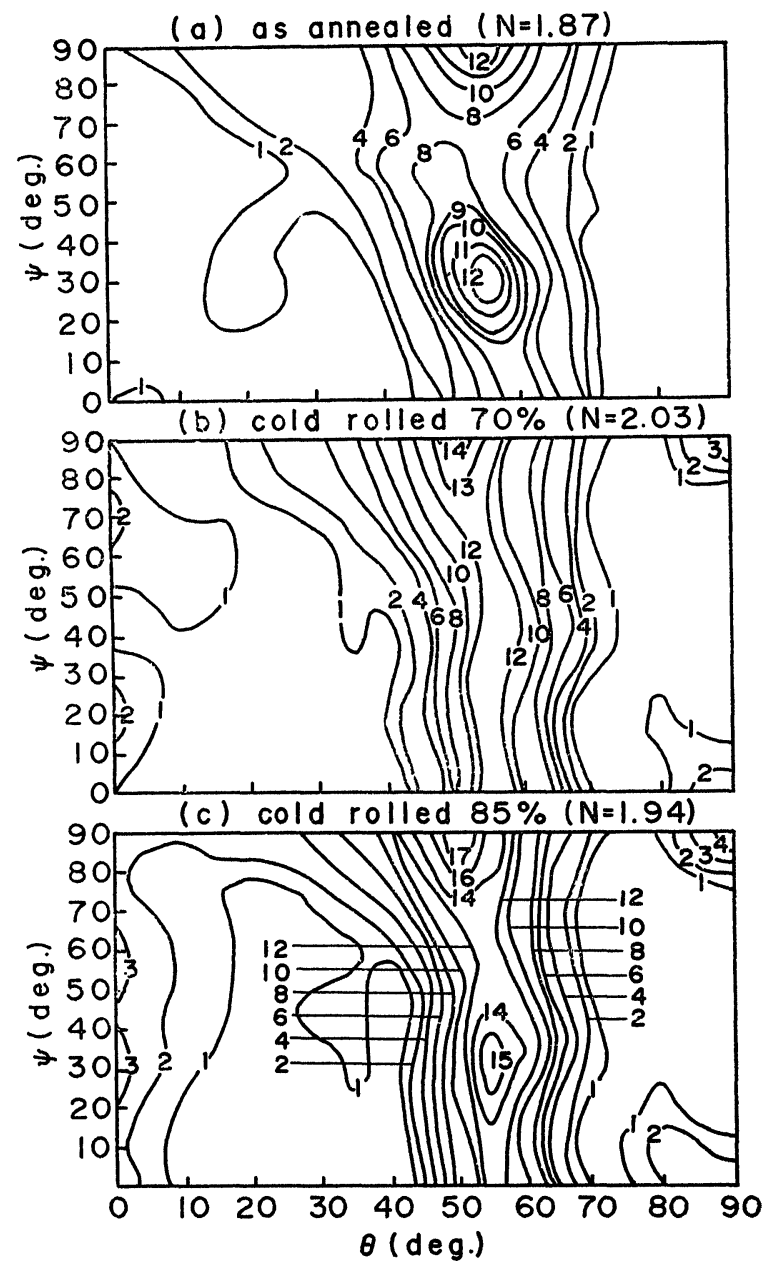

FIGURE $2 \phi=45$ deg sections showing the development of the rolling textures in the $\{111\}\langle 110\rangle$ specimen.

indicates that, although the $\{111\}\langle 110\rangle$ orientations are stable, a slight rotation takes place about the $\langle 110\rangle$ axes lying in the rolling direction.

Origins of the minor peaks at the $\{110\}\langle 110\rangle$, $\{110\}\langle 001\rangle$ and $\{001\}\langle 310\rangle(\theta=0 \mathrm{deg}, \psi=26.6$ and $63.4 \mathrm{deg}$ ) orientations in Figures 2(b) and (c) are not known. 
The $\{111\}\langle 112\rangle$ specimen Figures $3(\mathrm{a})$ and (b) are $\phi=45 \mathrm{deg}$ sections, showing the rolling textures of this specimen at 70 and 85 pct reductions, respectively.

At 70 pct reduction, the rolling texture may be described as consisting of the $\{111\}\langle 112\rangle$ initial orientations superposed on the sharp $\langle 111\rangle$ fiber texture having the $\langle 111\rangle$ axes in the sheet normal direction. It is to be noted that the $\langle 111\rangle$ fiber
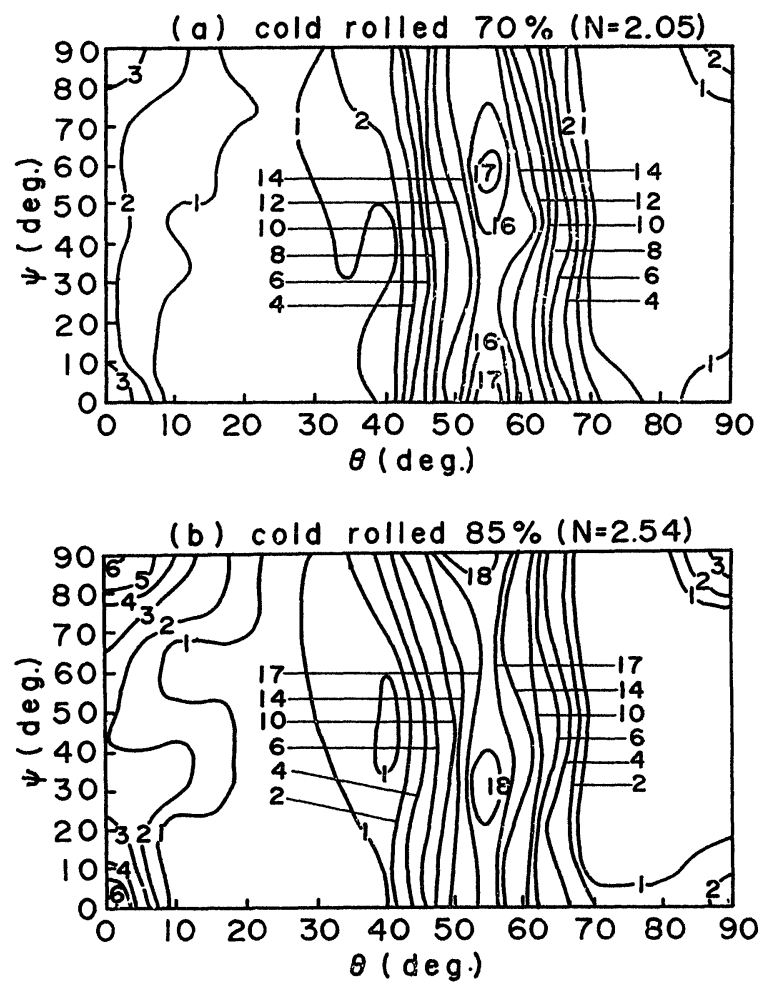

FIGURE $3 \phi=45$ deg sections of the crystallite orientation distribution, showing the rolling textures of the $\{111\}\langle 112\rangle$ specimen. (a) cold rolled 70 pct. (b) cold rolled 85 pct.

texture derived from the $\{111\}\langle 112\rangle$ initial texture, Figure 3(a), is much stronger than that derived from the $\{111\}\langle 110\rangle$ initial texture, Figure 2(b). As both the specimens were taken from the same original sheet and only the rolling directions were different, the sharpness of the initial textures must be the same in these two cases. In comparison with the $\{111\}\langle 110\rangle$ initial orientations, the $\{111\}$ $\langle 112\rangle$ initial orientations seem to be, therefore, more probable sources of the $\langle 111\rangle$ fiber texture. This fiber texture can be formed from the $\{111\}$ $\langle 112\rangle$ initial orientations by the rotation about the sheet plane normal.

At 85 pct reduction, the $\{111\}\langle 112\rangle$ component lost its prominence completely, while the $\langle 111\rangle$ fiber texture showed further development and the presence of the $\{111\}\langle 110\rangle$ component became distinct on the $\theta=55 \mathrm{deg}$ line, Figure 3(b). These orientation changes can be explained again in terms of rotation about the $\langle 111\rangle$ axes lying in the sheet normal direction; and the grains having the $\{111\}$ $\langle 112\rangle$ initial orientations appear to become more stable as the $\{111\}\langle 110\rangle$ orientations are approached as a result of this rotation.

Among the members of the $\langle 111\rangle$ fiber texture, the $\{111\}\langle 110\rangle$ orientations are most distant from the $\{111\}\langle 112\rangle$ orientations. If a further rotation were to take place about the $\langle 111\rangle$ fiber axes, then grains which have reached the $\{111\}\langle 110\rangle$ orientations would be brought back nearer to the $\{111\}$ $\langle 112\rangle$ initial orientations, thus becoming unstable again. From this reasoning, the rotation about the $\langle 111\rangle$ fiber axes must terminate at the $\{111\}\langle 110\rangle$ orientations.

As the $\{111\}\langle 110\rangle$ orientations are, at the same time, members of the $\langle 110\rangle$ fiber texture having the $\langle 110\rangle$ fiber axes in the rolling direction, they constitute the transition points of these fiber textures; and further rotations might be possible about the $\langle 110\rangle$ fiber axes lying in the rolling direction.

Taking these rotation paths into account, it would be expected that, at high reductions, the rolling texture of the $\{111\}\langle 112\rangle$ specimen approaches to that of the $\{111\}\langle 110\rangle$ specimen. In fact, it can be seen that a close similarity exists between Figure 2(c) and Figure 3(b), with the exception of the $\{001\}\langle 110\rangle$ component, which showed a remarkable development in the $\{111\}$ $\langle 112\rangle$ specimen.

Single crystals having the (111)[112] orientation are reported to be stable up to 70 pct reduction in thickness. ${ }^{1,25-27}$ Above 90 pct reduction, crystal rotation occurred about the [110] axis lying in the transverse direction, producing a weak $\{001\}$ $\langle 110\rangle$ texture. $^{26}$

The crystal rotation about the sheet plane normal was observed only under restricted conditions. Barrett and Levenson cold rolled (111)[112] single crystals under constraints up to 97 pct reduction in thickness and observed a large orientation spread about the sheet plane normal. ${ }^{1} \mathrm{Hu}$ and Cline observed such orientation spread at low reductions in the surface layers of a 2 pct $\mathrm{Al}-\mathrm{Fe}$ single crystal having the (111)[112] initial orientation. ${ }^{26}$ They 
ascribed this to the higher stress on the specimen surface due to friction between the rolls and the specimen.

Taking these results into account, it might be deduced that, in the present $\{111\}\langle 112\rangle$ polycrystalline specimen, the crystal rotation about the sheet plane normal would be caused by the constraining effect of the grain boundary. Such possibility appears to be indicated by Figure 4 , which shows (001) facet pits on the rolling plane of Steel 18 cold rolled 70 pct. It can readily be seen that, in a (111) grain, the pyramids rotate continuously about the sheet plane normal, as the grain boundary is approached.

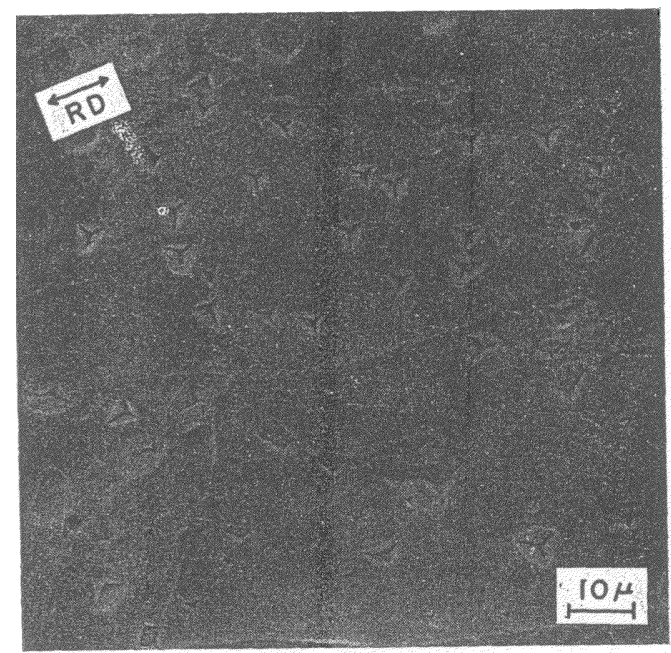

FIGURE 4 (100) facet pits on the rolling plane of Steel 18 rolled 70 pct. (The sample was polished to the center of the specimen thickness).

The $\{110\}\langle 001\rangle$ specimen Figures $5(\mathrm{a})$ to (f) are $\phi=45$ deg sections of the crystallite orientation, showing the textures of this specimen at each stage of cold rolling. Figure 5(a) represents a sharp initial texture. After 30 pct cold rolling, the maximum at the $\{110\}\langle 001\rangle$ orientation disappeared, while two broad peaks emerged at the positions corresponding to the $\{554\}\langle 225\rangle$ orientations, Figure $5(\mathrm{~b})$. At 50 pct reduction, these peaks started to leave the $\{554\}\langle 225\rangle$ orientation, aiming at the $\{111\}\langle 112\rangle$ orientations, Figure 5(c). At 70 pct reduction, these were reached exactly. Note that, in Figure 5(d), one of the main peaks is located at $\theta=55 \mathrm{deg}$ on the $\psi=0 \mathrm{deg}$ line. The height and sharpness of these peaks were remarkably increased.
Thus, most of the orientation changes up to 70 pct reduction occurred along the $\psi=0$ deg line and these would be described as a crystal rotation about the $\langle 110\rangle$ axes lying in the transverse direction.

On further cold rolling to a reduction of $85 \mathrm{pct}$, Figure 5(e), broad peaks at the $\{111\}\langle 112\rangle$ orientations developed into the $\langle 111\rangle$ fiber texture having the $\langle 111\rangle$ axes in the sheet normal direction. A new peak was formed now at about $\theta=47 \mathrm{deg}$ on the $\psi=90 \mathrm{deg}$ line, indicating the increase of the members of the $\langle 110\rangle$ fiber texture having the $\langle 110\rangle$ fiber axes in the rolling direction.

After 91 pct cold reduction, both this and the $\langle 111\rangle$ fiber texture increased their intensities, whereas a preference for the $\{111\}\langle 112\rangle$ orientation became less evident on the $\theta=55$ deg line, Figure 5(f).

Thus, the orientation changes above 70 pct reduction occurred predominantly on the $\theta=55$ deg line and on the $\psi=90 \mathrm{deg}$ line, and were essentially the same as those observed on the $\{111\}\langle 112\rangle$ and $\{111\}\langle 110\rangle$ specimens. Note that, aside from the presence of the $\{001\}\langle 110\rangle$ component and aside from the differences in the sharpness, Figures 2(c), 3 (b) and 5(f) represent qualitatively the same texture. It might be that, although starting points are different, the $\{110\}\langle 001\rangle,\{111\}\langle 112\rangle$ and $\{111\}$ $\langle 110\rangle$ specimens follow the same rotation path to reach the same stable end orientation. The rotation path seems to consist of three stages. At low reductions, rotations occur about the $\langle 110\rangle$ axes lying in the transverse direction; and grains having the initial orientations in the range $\{110\}\langle 001\rangle$ to $\{111\}\langle 112\rangle$ are brought into the $\{111\}\langle 112\rangle$ orientations. Thus, at medium reductions, a $\{111\}$ $\langle 112\rangle$ texture develops. Then rotations occur about the $\langle 111\rangle$ axes lying in the sheet normal direction. This leads to the formation of a $\langle 111\rangle$ fiber texture having the $\langle 111\rangle$ fiber axes in the sheet normal direction. Those grains which have arrived at the $\{111\}\langle 110\rangle$ orientations undergo additional rotation about the $\langle 110\rangle$ axes in the rolling direction.

Bennewitz ${ }^{4}$ also divided the development of the rolling textures into three stages, but in his case the rotation path is different. In his model, the most basic assumption consists of the presence of the $\langle 110\rangle 60 \mathrm{deg}$ RD fiber texture. Members of this fiber texture were, in the range $\phi=34$ to $45 \mathrm{deg}$, projected on the $\phi=45 \mathrm{deg}$ section and were shown in dashed lines in Figures 5(a) to (d). (Members lying in the range $(\overline{5} 45)[2 \overline{5} 2]$ to $(\overline{1} 12)$ [110] are located quite close to the $\phi=45 \mathrm{deg}$ 

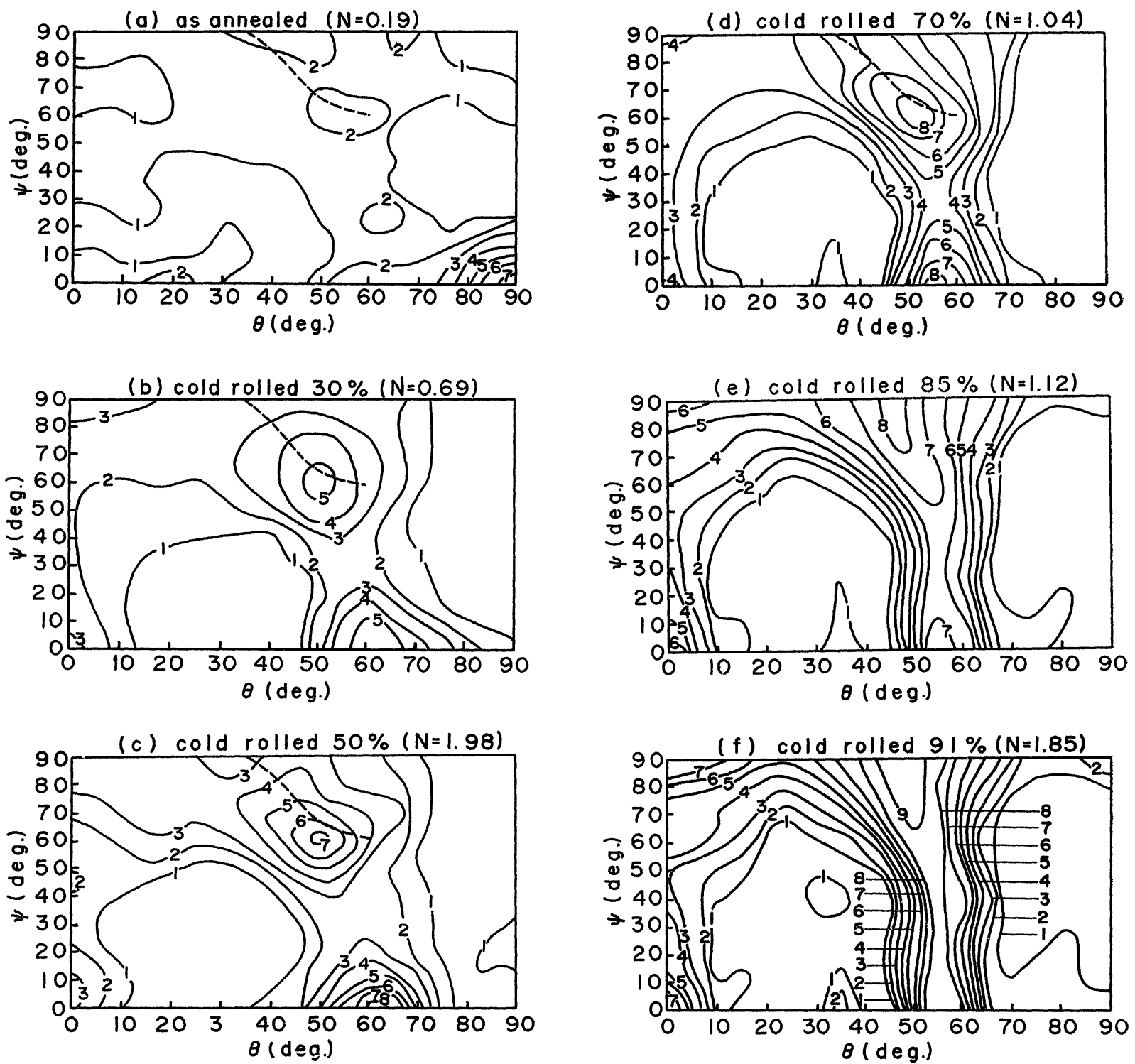

FIGURE $5 \phi=45 \mathrm{deg}$ sections of the crystallite orientation distribution, showing the rolling textures of the $\{110\}\langle 001\rangle$ specimen. (a) as annealed. (b) cold rolled 30 pct. (c) cold rolled 50 pct. (d) cold rolled 70 pct. (e) cold rolled 85 pct. (f) cold rolled 91 pct.

section.) If grains gather around the $\{554\}\langle 225\rangle$ orientations at low reductions and rotate further about the $\langle 110\rangle 60 \mathrm{deg} \mathrm{RD}$ fiber axes toward the $\{112\}\langle 110\rangle$ orientations, as Bennewitz predicted, then it should be observed in the present $\{110\}$ $\langle 001\rangle$ specimen, that one of the sharp maxima which were formed at the $\{554\}\langle 225\rangle$ orientations at 30 pct reduction would move with increasing reduction along this dashed line, aiming at the $\{112\}\langle 110\rangle$ orientations.

However, the observed orientation changes did not occur along the dashed line, but along the $\theta=55 \mathrm{deg}$ line, as described above. In addition, it was noted earlier, in Figure 5(d), that the centers of the maxima arrived exactly at the $\{111\}\langle 112\rangle$ orientations, passing through the $\{554\}\langle 225\rangle$ 
orientations. Thus, the significance of specifying the $\{554\}\langle 225\rangle$ orientations seems to be doubtful in the case of the rolling texture.

The process occurring in the present $\{110\}\langle 001\rangle$ specimen below 70 pct reduction is qualitatively the same as those observed on the single crystals having this orientation. ${ }^{27,30}$ In the present polycrystalline specimen, however, the $\{001\}\langle 110\rangle$ component showed a remarkable development above 85 pct reduction. The same phenomenon was observed also in the $\{111\}\langle 112\rangle$ specimen cold rolled $85 \mathrm{pct}$, Figure 3(b). This suggests again that the rotation paths are partly common in both cases.

The $\{110\}\langle 110\rangle$ specimen Figures $6(\mathrm{a})$ to (d) are $\phi=45 \mathrm{deg}$ sections of the crystallite orientation distribution, showing the development of the rolling textures in this specimen. Figure 6(a) shows the $\{110\}\langle 110\rangle$ initial texture.

After 30 pct cold reduction, the sharpness of the initial texture decreased and minor peaks appeared at the $\{001\}\langle 110\rangle,\{001\}\langle 100\rangle,\{111\}\langle 110\rangle$ and $\{111\}\langle 112\rangle$ orienations. However, in a marked contrast to the $\{110\}\langle 001\rangle$ specimen, large amounts
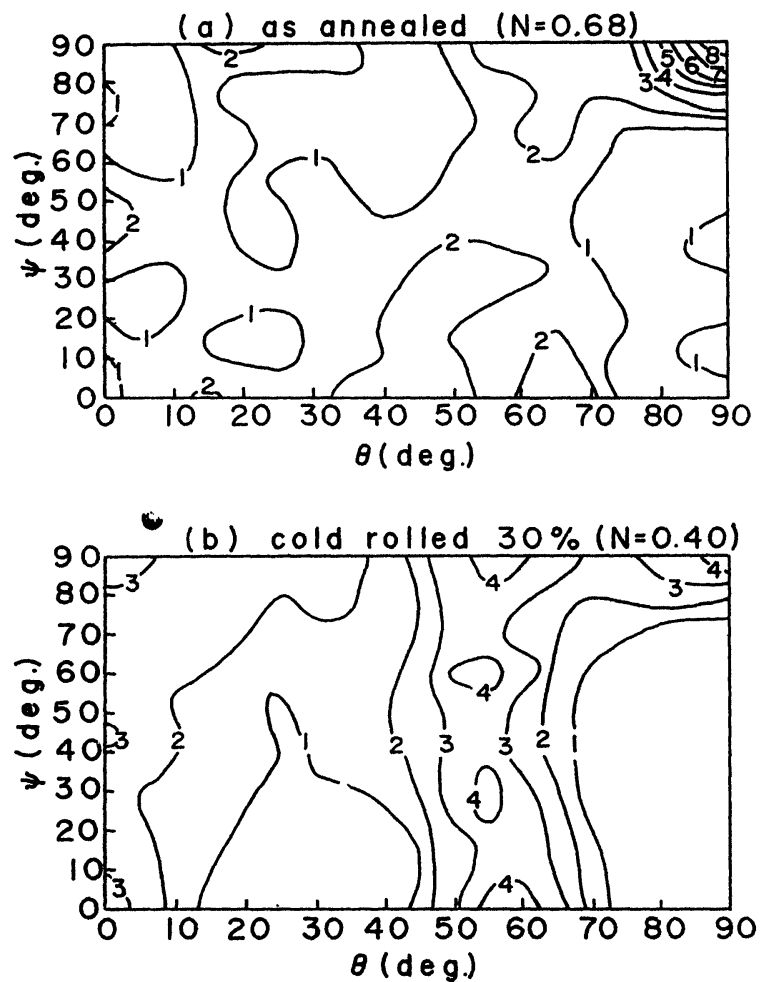

of the crystals retained their initial orientations, showing their high stabilities. A continuous orientation spread was produced on the $\psi=90 \mathrm{deg}$ line between the $\{111\}\langle 110\rangle$ and $\{110\}\langle 110\rangle$ orientations, Figure 6(b).

At 50 pct reduction, the original peak at the $\{110\}\langle 110\rangle$ orientations disappeared completely and, instead, weak $\langle 111\rangle$ and $\langle 100\rangle$ fiber textures, having their fiber axes in the sheet normal direction, developed, Figure 6(c).

After 70 pct cold rolling, a broad peak was formed on the $\psi=90 \mathrm{deg}$ line. This peak extended preferentially along the $\theta=50$ deg line, Figure 6(d).

Of these orientation changes, those along the $\psi=90 \mathrm{deg}$ line are described as a rotation about the $\langle 110\rangle$ axes in the rolling direction. As a result of this rotation, grains having the $\{110\}\langle 110\rangle$ initial orientations could thus be brought as far as $\theta=50$ deg on the $\psi=90$ deg line after 70 pct reduction. Although single crystals having the (110)[110] orientations tend also to rotate slightly about the rolling direction, they retain their initial orientations up to 70 pct reduction. ${ }^{27,29}$ Taking these results into account, it might be concluded that this
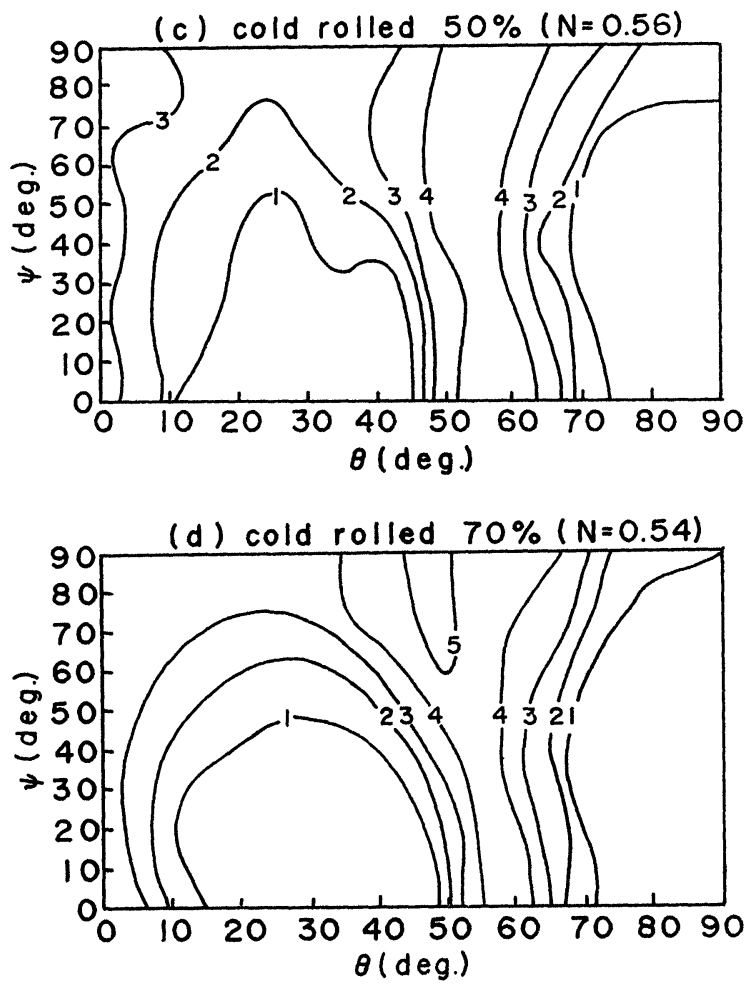

FIGURE $6 \phi=45 \mathrm{deg}$ sections of the crystallite orientation distribution, showing the rolling textures of the $\{110\}$ $\langle 110\rangle$ specimen. (a) as annealed. (b) cold rolled 30 pct. (c) cold rolled 50 pct. (d) cold rolled 70 pct. 
rotation occurs more readily in polycrystals due to the constraining effect of the grain boundary.

The $\{001\}\langle 110\rangle$ specimen Figures $7(a)$ and (b) are $\phi=45$ deg sections of the crystallite orientation distribution, showing the textures of this specimen before and after 70 pct cold rolling. The initial texture contained a large orientation spread along the $\psi=90 \mathrm{deg}$ line, Figure 7(a). After
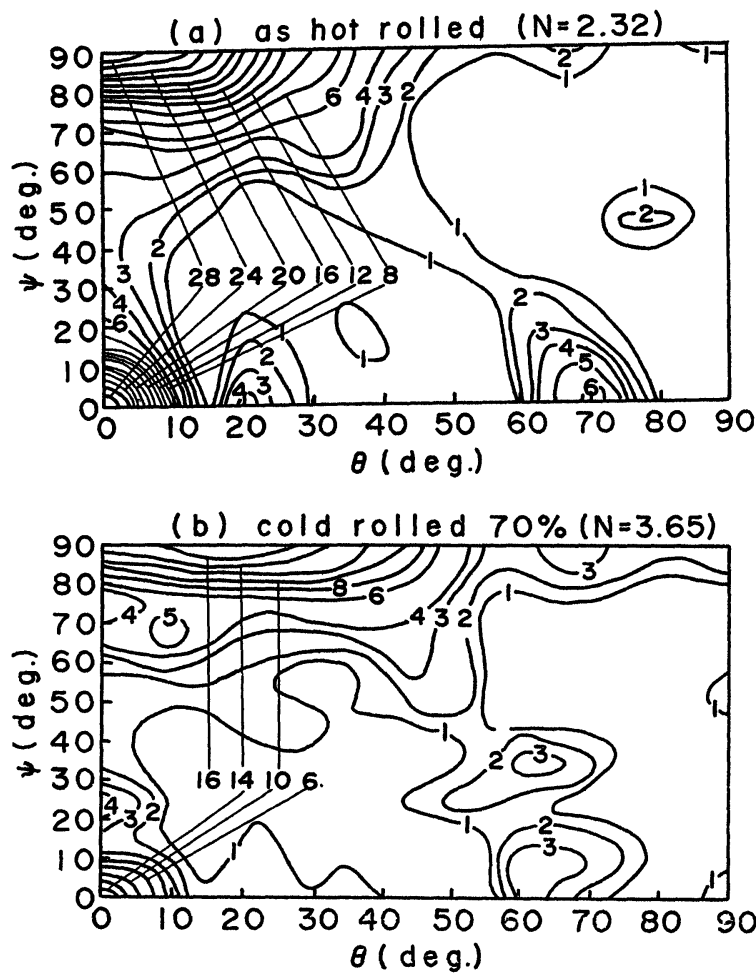

FIGURE $7 \phi=45$ deg sections of the crystallite orientation distribution, showing the textures of the $\{001\}\langle 110\rangle$ specimen before and after 70 pct cold rolling. (a) as hot rolled. (b) cold rolled 70 pct.

70 pct reduction, the $\{001\}\langle 110\rangle$ orientations decreased remarkably and the broad peak on the $\psi=90 \mathrm{deg}$ line was shifted along this line to the $\{114\}\langle 110\rangle$ orientations, Figure 7(b). Further cold rolling up to $90 \mathrm{pct}$ produced no substantial change in the rolling texture.

Thus, in this specimen, grains rotate at lower reductions about the $\langle 110\rangle$ axes in the rolling direction; and above 70 pct reduction, they are stable.

The instability of the $\{001\}\langle 110\rangle$ initial texture was also confirmed by Richards and Ormay ${ }^{9}$ in a
1.5 pct Si steel. As single crystals having the (001) [110] orientation are generally stable, ${ }^{25,27}$ this instability might be attributed to the constraining effect of the grain boundary.

If this explanation is correct, and if the results of the $\{111\}\langle 112\rangle$ and $\{110\}\langle 110\rangle$ specimens are taken into account, it would be concluded that this constraining effect enhances crystal rotations, which would not be expected to take place in single crystals. This might be possible in $\alpha-\mathrm{Fe}$, in which slip can occur on any of the $\{110\},\{112\}$ and $\{123\}$ planes. In this case, it would be expected that the constraining effect of the grain boundary could be relaxed relatively easily, accompanying large orientation changes in the neighbourhood of the grain boundary.

Aspden $^{31}$ studied the effect of constraints in 3 pct $\mathrm{Si}-\mathrm{Fe}$ crystals having the (001)[100] initial orientation. He found that the amount of rotation toward the $\{001\}\langle 110\rangle$ stable end orientations was decreased by using constraints during rolling.

Thus, this effect seems to depend on the orientation of the grain in question. Furthermore, it may be influenced by the shape, grain size and orientation of neighbouring grains. At present, this problem is too difficult to be discussed theoretically.

Low-carbon steel Figures 8(a) to (f) are $\phi=45 \mathrm{deg}$ sections, showing the development of the rolling textures in Steel 13.

The texture of the starting hot-band contained weak components such as $\{001\}\langle 110\rangle,\{221\}$ $\langle 114\rangle(\theta=70.5 \mathrm{deg}, \psi=0 \mathrm{deg})$ and $\{221\}\langle 110\rangle$ $(\theta=70.5 \mathrm{deg}, \psi=90 \mathrm{deg})$, Figure 8(a).

After 15 pct reduction, a distinct peak was formed at the $\{332\}\langle 113\rangle(\theta=64.8 \mathrm{deg}, \psi=0 \mathrm{deg})$ orientations. The $\{001\}\langle 110\rangle$ orientations also sharpened somewhat, while other orientations on the $\theta=0 \mathrm{deg}$ line decreased, Figure $8(\mathrm{~b})$. These orientation changes may be explained in terms of the observations of Koh and Dunn. ${ }^{25}$ Their results on single crystals having a [110] axis in the transverse direction indicate that grains having an initial orientation in the range $\{110\}\langle 001\rangle$ to $\{112\}\langle 111\rangle$ tended to rotate to the $\{111\}\langle 112\rangle$ orientations, while those in the range $\{112\}\langle 111\rangle$ to $\{001\}\langle 110\rangle$ rotated to the $\{001\}\langle 110\rangle$ orientations.

On the $\psi=90 \mathrm{deg}$ line, a broad peak was formed at about $\theta=10 \mathrm{deg}$. As was already shown in Figure $7(\mathrm{~b})$, this neak might be derived from the $\{001\}\langle 110\rangle$ initial orientations by the rotation about the $\langle 110\rangle$ axes in the rolling direction. This 

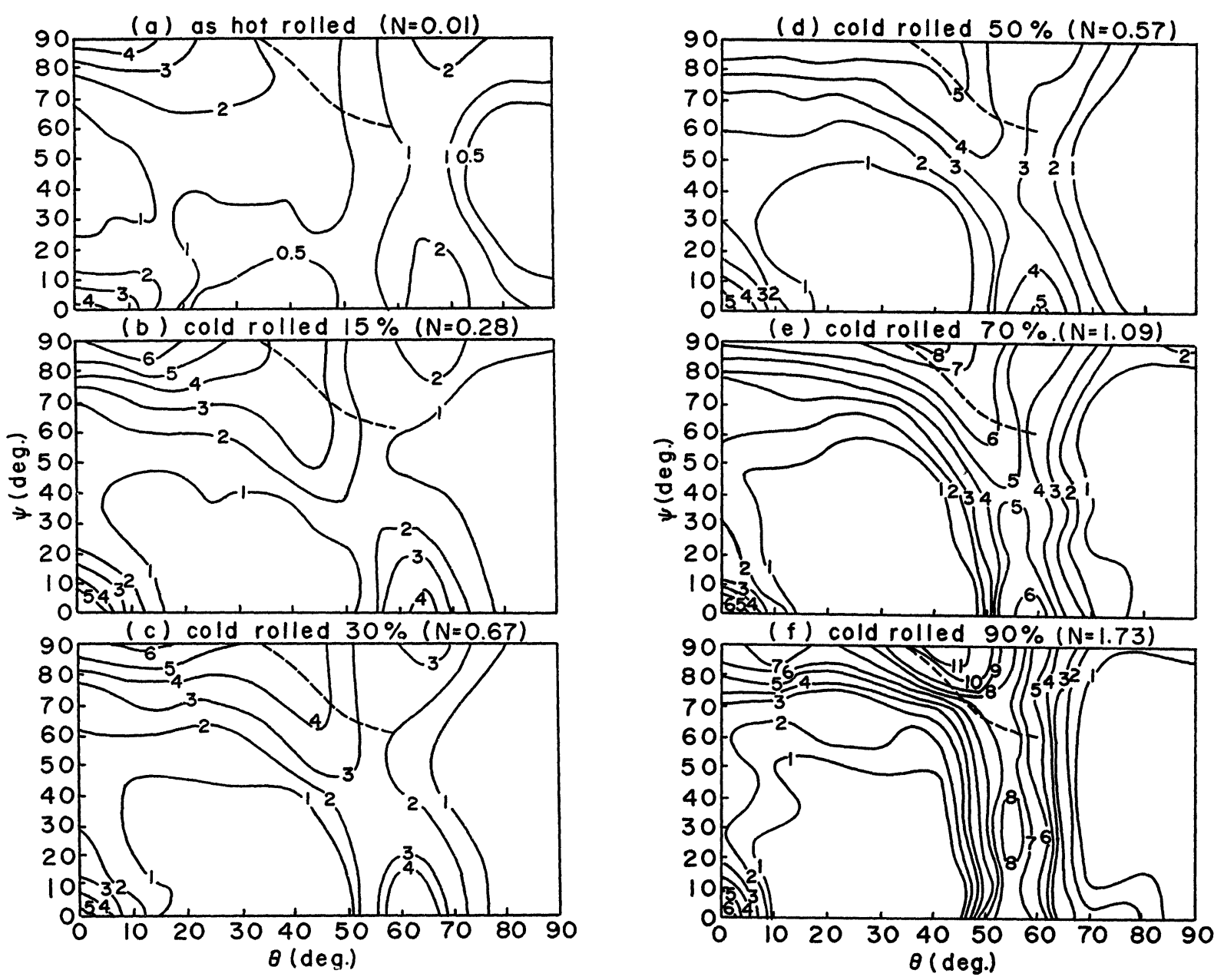

FIGURE $8 \phi=45 \mathrm{deg}$ sections, showing the development of the rolling textures in an aluminum killed steel.

may explain the fact that the increase of the $\{001\}\langle 110\rangle$ orientations after 15 pct cold rolling was relatively small. From this broad peak, contour lines were bulging to the center of the $\phi=45 \mathrm{deg}$ section, forming a ridge.

At 30 pct reduction, the $\{554\}\langle 225\rangle$ orientations became one of the main components of the rolling texture, Figure 8(c). Comparing Figure 8(c) with Figure 1, it would suggest that the ridge, which extended to the center of $\phi=45$ deg section, was associated also with the $\{554\}\langle 225\rangle$ orientations. At the beginning of this stage, grains having the initial orientations in the range $\{110\}\langle 110\rangle$ to $\{221\}\langle 110\rangle$ began to rotate about the $\langle 110\rangle$ axes jn the rolling direction, producing a small peak at the $\{332\}\langle 110\rangle$ orientations $(\theta=64.8 \mathrm{deg}, \psi=$ $90 \mathrm{deg})$.

At 50 pct reduction, this crystal rotation proceeded further and the $\{332\}\langle 110\rangle$ peak merged into the ridge which ran obliquely across the $\phi=45 \mathrm{deg}$ section, Figure 8(d). On the other hand, the broad peak, which had been located at $\theta=10 \mathrm{deg}$ on the $\psi=90 \mathrm{deg}$ line collapsed and extended into a plateau running parallel to the $\psi=90 \mathrm{deg}$ line. In this way, two crystal rotations were occurring on the $\psi=90$ deg line in the opposite directions.

At 70 pct reduction, a sharp maximum was formed at the $\{335\}\langle 110\rangle$ orientations $(\theta=40 \mathrm{deg}$, $\psi=90 \mathrm{deg}$ ), Figure 8(e). This might be due to the superposition of these two rotations occurring 

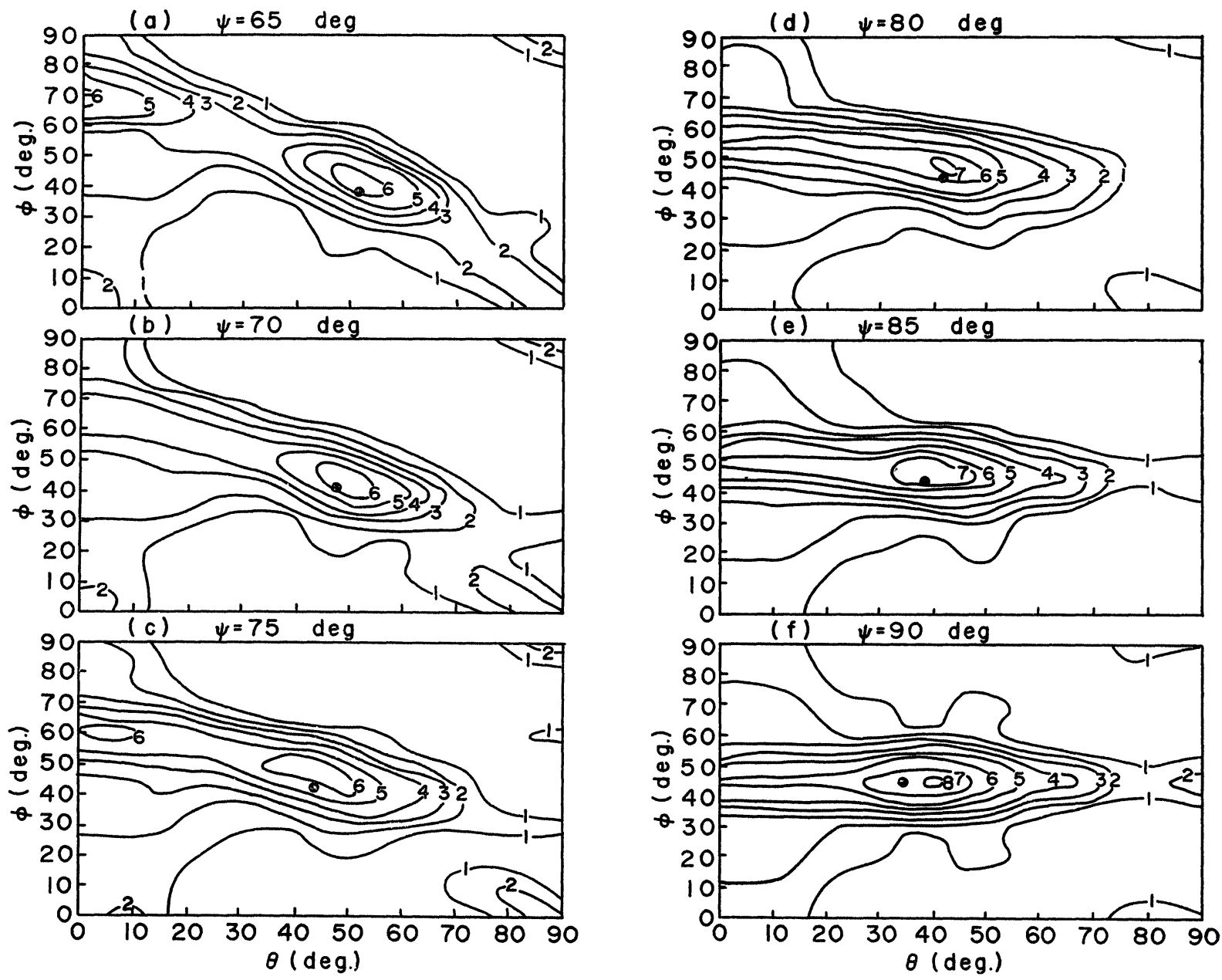

FIGURE 9 Constant $\psi$ sections of the crystallite orientaion distribution for an aluminum killed steel rolled 70 pct.

along the $\psi=90$ deg line. On the $\psi=0$ deg line, the peak at the $\{554\}\langle 225\rangle$ orientations tended to leave these orientations behind, and the presence of the $\langle 111\rangle$ fiber texture having the $\langle 111\rangle$ axes in the sheet normal direction became evident.

At 90 pct reduction, both the $\{335\}\langle 110\rangle$ peak and the $\langle 111\rangle$ fiber texture developed remarkably, Figure 8(f). In the latter, some preference for the $\{111\}\langle 110\rangle$ orientations seems to be present. The $\{554\}\langle 225\rangle$ orientations were no more at the center of the peak on the $\psi=0$ deg line.

Thus, the rolling textures of this specimen having a weak initial texture could be explained in terms of the crystal rotations which were observed in specimens having sharp initial textures. It can be constructed approximately by the superpositions of various components, which follow their predetermined rotation path to reach their stable end orientations.

Dashed lines in Figures 8(a) to (f) have the same meaning as those in Figures 5(a) to (d). Also in this case, the texture development did not proceed along these lines. With increasing cold reduction, the dashed line seemed to be rather traversed by the ridge running diagonally across the $\phi=45 \mathrm{deg}$ section. At higher reductions, this ridge became more vertical, and this is just the process through which the $\langle 111\rangle$ fiber texture having the $\langle 111\rangle$ axes in the sheet normal direction becomes more complete.

Bennewitz's model was examined from another viewpoint. Figures 9 (a) to (d) are the constant 
$\psi$ sections of the crystallite orientation distribution of Steel 13 cold rolled 70 pct. For the present purpose, sections in the range $\psi=65$ to $90 \mathrm{deg}$ are sufficient. Members of the $\langle 110\rangle 60 \mathrm{deg}$ RD fiber texture in the range $\{554\}\langle 225\rangle$ to $\{112\}\langle 110\rangle$ are contained in these sections and they are shown by black dots in the figures. Although a black dot was situated always near the center of the peak at each constant $\psi$ sections, the deviation between them was sometimes larger than the experimental error. The disagreement became largest at the $\psi=90 \mathrm{deg}$ section at which the crystallite orientation distribution exhibited a maximum. At other reductions, the skeleton line ${ }^{14}$ deviated from the members of the $\langle 110\rangle 60 \mathrm{deg}$ RD fiber texture much more definitely.

From these observations it can be concluded that the whole features of the orientation changes occurring continuously during cold rolling cannot be properly described in terms of the $\langle 110\rangle 60 \mathrm{deg}$ RD fiber texture.

The description in terms of the $\langle 111\rangle$ fiber texture having the $\langle 111\rangle$ axes in the sheet normal direction seems to be a better one. The same conclusion was reached by Takechi, Kato and Nagashima, ${ }^{8}$ Bunge ${ }^{16}$ and Heckler and Granzow. ${ }^{24}$ In the latter two investigations, the crystallite orientation distribution analysis was adopted.

\section{CONCLUSION}

The development of the rolling textures in lowcarbon steels having sharp initial textures was studied by using the crystallite orientation distribution analysis. Based on the result of this analysis, the rotation path of a grain having a specific initial orientation in a random polycrystal was deduced.

Grains having the $\{110\}\langle 001\rangle$ initial orientations rotate at low reductions about their $\langle 110\rangle$ axes in the transverse direction and reach the $\{111\}\langle 112\rangle$ orientations. Subsequently, they rotate about the $\langle 111\rangle$ axes in the sheet normal direction, forming a $\langle 111\rangle$ fiber texture. This rotation terminates, however, at the $\{111\}\langle 110\rangle$ orientations. Since these orientations are also members of the fiber texture having the $\langle 110\rangle$ axes in the rolling direction, grains rotate then about these $\langle 110\rangle$ axes, aiming at the $\{112\}\langle 110\rangle$ orientations.

Although starting points are different, grains having either $\{111\}\langle 112\rangle$ or $\{111\}\langle 110\rangle$ orientations seem to follow the same rotation path. The rotation about the $\langle 111\rangle$ axes in the sheet normal direction, mentioned above, would be caused by the constraining effect of the grain boundary.

Although single crystals having a [110] axis in the rolling direction are generally stable, grains having either $\{110\}\langle 110\rangle$ or $\{001\}\langle 110\rangle$ orientations in polycrystals are not stable and rotate about the $\langle 110\rangle$ axes lying in the rolling direction. This instability might be also attributed to the constraining effect of the grain boundary.

Thus, the rolling texture of ploycrystalline iron seems to be largely influenced by the constraining effect of the grain boundary. This may be due to the fact that, in $\alpha-F e$, slip can occur on any of the $\{110\},\{112\}$ and $\{123\}$ planes, so that the constraining effect of the grain boundary would be relaxed relatively easily. In this case large orientation changes would be produced in the neighbourhood of the grain boundary.

The development of the rolling texture in low carbon steels could not adequately be described in terms of Bennewitz's $\langle 110\rangle 60 \mathrm{deg}$ RD fiber texture.

\section{ACKNOWLEDGMENTS}

The authors wish to express their gratitude to Dr. S. Nagashima and Mr. S. Tani, Fundamental Research Laboratories of Nippon Steel Corp., for helpful discussions and criticisms, and to Dr. H. Kubotera for his encouragement during the course of this study.

\section{REFERENCES}

1. C. S. Barrett and L. H. Levenson, AIME Trans. 145, $281(1941)$.

2. F. Haessner and H. Weik, Arch. Eisenhüttenw. 27, 153 (1956).

3. H. Möller and H. Stäblein, Arch. Eisenhüttenw. 29, 377 (1958).

4. J. Bennewitz, Arch. Eisenhüttenw. 33, 393 (1962).

5. I. L. Dillamore and W. T. Roberts, Acta Met. 12, 281 (1964).

6. C. A. Stickles, Trans. Met. Soc. AIME 233, 1550 (1965).

7. R. A. Vandermeer and J. C. Ogle, Trans. Met. Soc. AIME 242, 1317 (1968).

8. H. Takechi, H. Kato and S. Nagashima, Trans. Met. Soc. AIME 242, 56 (1968).

9. P. N. Richards and M. K. Ormay, Trans. Met. Soc. AIME 245, 715 (1969).

10. R. J. Roe and W. R. Kriegbaum, J. Chem. Phys. 40, 2608 (1964).

11. R. J. Roe and W. R. Kriegba ım, J. Chem. Phys., 41, 737 (1964).

12. R. J. Roe, J. Appl. Phys., 36, 2024 (1965).

13. R. J. Roe, J. Appl. Phys. 37, 2069 (1966).

14. H. J. Bunge, Mathematische Methoden der Texturanalyse (Akademie Verlag, Berlin, 1969).

15. H. J. Bunge and F. Haessner, J. Appl. Phys. 39, 5503 (1968)

16. H. J. Bunge, Phys. Stat. Sol. 26, 167 (1968). 
17. H. J. Bunge, Kristall und Technik 3, 439 (1968).

18. H. J. Bunge and W. T. Roberts, J. Appl. Cryst. 2, 116 (1969).

19. T. Taoka, E. Furubayashi and S. Takeuchi, Japanese J. Appl. Phys. 4, 120 (1965).

20. B. F. Decker, E. T. Asp and D. Harker, J. Appl. Phys. 19, $388(1948)$.

21. L. G. Schulz, J. Appl. Phys. 20, 1030 (1948).

22. J. Grewen, D. Sauer and H. P. Wahl, Scripta Met. 3, 53 (1965).

23. P. R. Morris and A. J. Heckler, in Advances in X-Ray Analysis, (Plenum Press, New York, 1968), Vol. II, p. 454.
24. A. J. Heckler and W. G. Grazow, Met. Trans. 1, 2089 (1970).

25. P. K. Koh and C. G. Dunn, AIME Trans. 203, 401 (1955).

26. H. Hu and R. S. Cline, Trans. Met. Soc. AIME 224, 784 (1962).

27. T. Taoka, E. Furubayashi and S. Takeuchi, Trans. Iron Steel Inst. Japan 6, 290 (1966).

28. C. G. Dunn, Acta Met. 2, 173 (1954).

29. J. L. Walter and W. R. Hibbard, Jr., Trans. Met. Soc. AIME 212, 731 (1958).

30. H. Hu, Trans. Met. Soc. AIME, 221, 130 (1951).

31. R. G. Aspden, Trans. Met. Soc. AIME 215, 986 (1959). 